\title{
Hyperimmune plasma infusion in an immunocompromised Covid-19 patient previously treated for follicular lymphoma
}

\author{
Guido Levi $^{1}$, Chiara Rocchetti ${ }^{1}$, Roberto Magri ${ }^{1}$, Silvia Uccelli ${ }^{1}$, Damiano Bottone ${ }^{1}$, Federico Quadri ${ }^{1}$, \\ Mauro Novali ${ }^{1}$, Alessandro D. Santin ${ }^{2}$, Michela Bezzi ${ }^{1}$ \\ ${ }^{1}$ Interventional Pulmonology Department, ASST Spedali Civili, Brescia, Italy; ${ }^{2}$ Department of Obstetrics and \\ Gynecology, Yale University School of Medicine, New Haven, CT, USA
}

\begin{abstract}
Covid-19 in immunocompromised patients shows a prolonged course and may lead to a poor prognosis. Although data on hyperimmune plasma for treatment of Covid-19 suggest an improved outcome in immunocompetent patients, limited data are currently available in immunocompromised patients. We present the case of a 62-year-old Caucasian woman, who was previously treated with obinutuzumab and bendamustine for follicular lymphoma and showed a prolonged positive test for Covid-19. Since no improvement was observed with standard of care (including remdesivir),
\end{abstract}

Correspondence: Dr. Guido Levi, Interventional Pulmonology department, ASST Spedali Civili, P.le Spedali Civili 1, 25123 Brescia, Italy. Tel. +39.030. 3995593- Fax +39.030.3995076.

E-mail guido.levi@yahoo.it

Key words: Covid-19; hyperimmune plasma; case report; lymphoma; cancer.

Conflicts of interest: The authors declare that they have no conflict of interest.

Contributions: All authors contributed to the study conception and design. Material preparation, data collection and analysis were performed by GL and CR; the first draft of the manuscript was written by $\mathrm{GL}$ and $\mathrm{CR}$, and all authors commented on previous versions of the manuscript. All authors read and approved the final manuscript and agreed to be accountable for all aspects of the work.

Ethics approval: No ethical committee approval was required for this case report by the Department, because this article does not contain any studies with human participants or animals

Consent for publication: The patient has consented to the submission of the case report to the journal.

Received for publication: 27 March 2021.

Accepted for publication: 12 May 2021.

${ }^{\circ}$ Copyright: the Author(s), 2021

Licensee PAGEPress, Italy

Monaldi Archives for Chest Disease 2021; 91:1867

doi: 10.4081/monaldi.2021.1867

This article is distributed under the terms of the Creative Commons Attribution Noncommercial License (by-nc 4.0) which permits any noncommercial use, distribution, and reproduction in any medium, provided the original author(s) and source are credited. the possibility of hyperimmune plasma infusion was discussed. A first dose of hyperimmune plasma was administered, with subsequent onset of fever, increasing inflammatory indexes and worsening radiological findings. Three days later a second dose of plasma was administered. Within twelve hours cough and fever disappeared, and oxygen at rest was discontinued. The patient was discharged 5 days later, and nasopharyngeal swabs resulted negative 16 days after discharge.

\section{Introduction}

Since its first outbreak in Hubei, China, in December 2019, severe acute respiratory syndrome coronavirus-2 (SARS-CoV-2) has rapidly spread all over the world [1]. The clinical course ranges from asymptomatic infection to severe pneumonia and acute respiratory distress syndrome [2]. Recent studies demonstrated that some subgroups of patients suffering from Coronavirus Disease 19 (Covid-19) may experience more severe outcomes than other populations [3]. Moreover, it has been observed that immunocompromised patients may have a prolonged course of the disease [4].

We present the case of a 62-year-old Caucasian woman infected with Covid-19, who had been previously treated with chemotherapy for follicular lymphoma. She received standard of care treatment (enoxaparin, dexamethasone) for Covid-19, including remdesivir, without significant clinical improvement. After the administration of convalescent plasma, we observed immediate clinical improvement. Repeated nasopharyngeal swabs (NPS) tested positive for two months.

\section{Case Report}

A 62-year-old Caucasian woman was admitted to our hospital due to dyspnea, dry cough and worsening asthenia.

She had a significant history of hematologic cancer (stage IV follicular lymphoma in remission, treated with obinutuzumab and bendamustine in 2019), diverticulosis and a Body Mass Index in the overweight range $\left(28.9 \mathrm{~kg} / \mathrm{m}^{2}\right)$. Allergy to ceftriaxone and ciprofloxacin was also reported.

Twenty-six days earlier a NPS for Covid-19 had been performed due to anosmia and the virus infection was detected; she was treated at home with vitamin C and vitamin D3 supplements since then. Nine days before admission the patient had been hospitalized for $48 \mathrm{~h}$ due to persistent cough and asthenia, and a diag- 
nosis of Covid-19 bilateral interstitial pneumonia was obtained at CT scan with less than $25 \%$ parenchymal involvement. During these $48 \mathrm{~h}$ dexamethasone $6 \mathrm{mg}$ qd was administered intravenously and rapidly tapered because of the advent of fever for two nights. Prophylaxis with oral trimethoprim/sulfamethoxazole and itraconazole was then initiated together with subcutaneous enoxaparin 4000 IU qd. The patient was discharged since no respiratory failure was observed.
At the time of readmission peripheral oxygen saturation at room air was $89 \%$, and mild respiratory failure was observed at the arterial blood gas analysis, therefore oxygen was promptly administered. Physical examination showed multiple oral and ocular aphthae and bilateral velcro crackles, with no other significant findings. The chest CT scan showed no signs of pulmonary embolism, confirming bilateral interstitial pneumonia with $40 \%$ lung involvement (Figure 1).
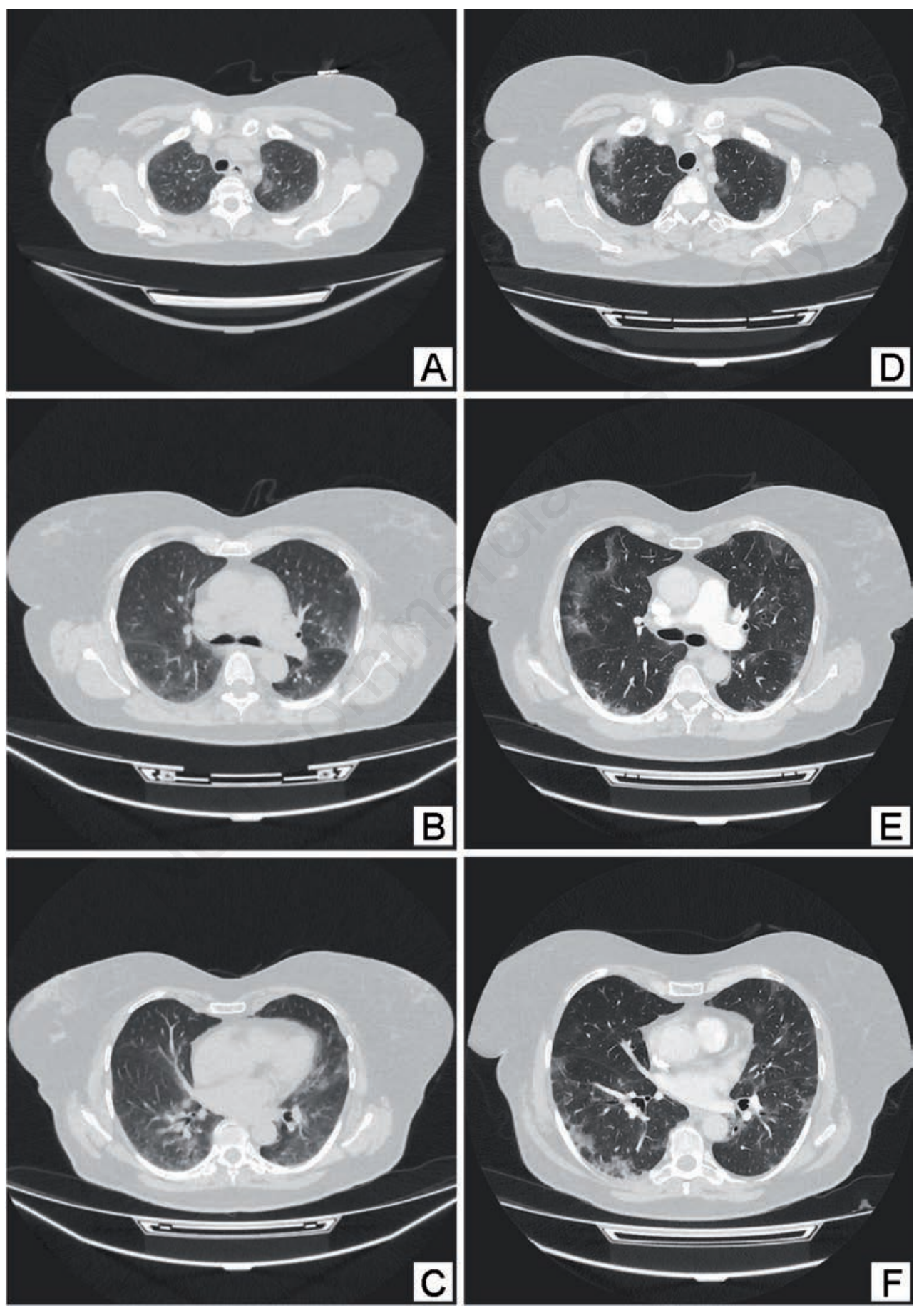

Figure 1. Chest CT-scan at first admission (day -9, left side) and at second admission (day 0, right side), at three different levels: A) and D) upper lobes; B) and E) carina; C) and F) lower lobes. Please note that two different machines were used. 
Blood tests detected lymphopenia (lymphocytes 820 cells/uL, normal values 900-4000), elevated C-reactive protein $(133.1 \mathrm{mg} / \mathrm{L}$, n.v. $<5)$, lactate dehydrogenase (470 U/L, n.v. 135225), fibrinogen (631 U/L, n.v. 170-420) and ferritin (1391 ug/L, n.v. 13-150). The complete absence of CD19 B lymphocytes (n.v. 86-648 cell/uL) and low CD4 T lymphocytes (99 cell/uL, n.v. 273-1852 cell/uL) due to recent chemotherapy were confirmed. The main laboratory abnormalities and their trends are shown in Figure 2.

Culture tests for fungi and multiple viruses (Adenovirus, Herpes Virus 1 and 2) on a swab from the aphthae and blood cultures for bacteria, fungi, Cytomegalovirus, Adenovirus, HHV6 and HHV8 resulted negative, despite the low number of CD4 T lymphocytes (below the safety cutoff of 500 cells $/ \mathrm{uL}$ ). Itraconazole, trimethoprim/sulfamethoxazole and valaciclovir were potentiated as a prophylactic measure.

Ten days later $\left(36^{\text {th }}\right.$ day from the first positive NPS for Covid-19), the arterial blood gas analysis still showed mild respiratory failure at rest, with severe dyspnea and oxygen desaturation during efforts, therefore High-Flow nasal cannula and pronation cycles were introduced. Culture tests on the bronchoalveolar lavage were negative. No antibodies against SARS-CoV2 were detected at the serological test $(0.1$, cutoff index negative $<1.0)$.

The case was thoroughly and continuously discussed with our infectious disease specialists and hematologists, who agreed to introduce remdesivir. Since no significant clinical and/or radiological improvement was observed, the possibility of hyperimmune plasma infusion was discussed, although this therapy is generally recommended within the third day after the admission or within the seventh day from symptoms' onset. The local Ethical Committee approved the indication and the patient signed the informed consent.
After 42 days from the first positive NPS for Covid-19 a first dose of hyperimmune plasma (500 cc, titer 1:160, negative value $<1: 10$ ) was administered with subsequent onset of fever and progressively increasing inflammatory indexes; during the following hours worsening radiological findings at chest $\mathrm{x}$-ray were also observed; in 3 days C-reactive protein decreased and, as per protocol, a second infusion of plasma (500 cc, titer 1:80, negative value $<1: 10)$ was administered 4 days later; inflammatory indexes were monitored every 6 hours after each infusion and reduction in CRP continued. Remdesivir was continued for a total of 10 days. Twelve hours after the second infusion of hyperimmune plasma, cough and fever disappeared. HFNC was progressively reduced up to discontinuation, but exercise desaturation was still observed. Antibodies against SARS-CoV2 were detected at the serological test $(3.32$, cutoff index negative $<1.0$ ). The patient was discharged 5 days after the second plasma infusion, with only mild hypoxemia, no cough and no dyspnea at rest. Oxygen during efforts was prescribed. The NPS for Covid-19 was still positive.

Patient was followed up at home through several visits, and she immediately began respiratory physiotherapy, reporting progressive clinical improvement up to the discontinuation of oxygen therapy. After 16 days (66 days after the first positive NPS for Covid-19) the NPS finally resulted negative. A complete timeline of the events is available in Figure 3.

\section{Discussion}

Innate and adaptive immune responses are fundamental to defeat viral infections. Accordingly, clinical status and prognosis of Covid19 in immunosuppressed patients may be poor [2]. A multicenter
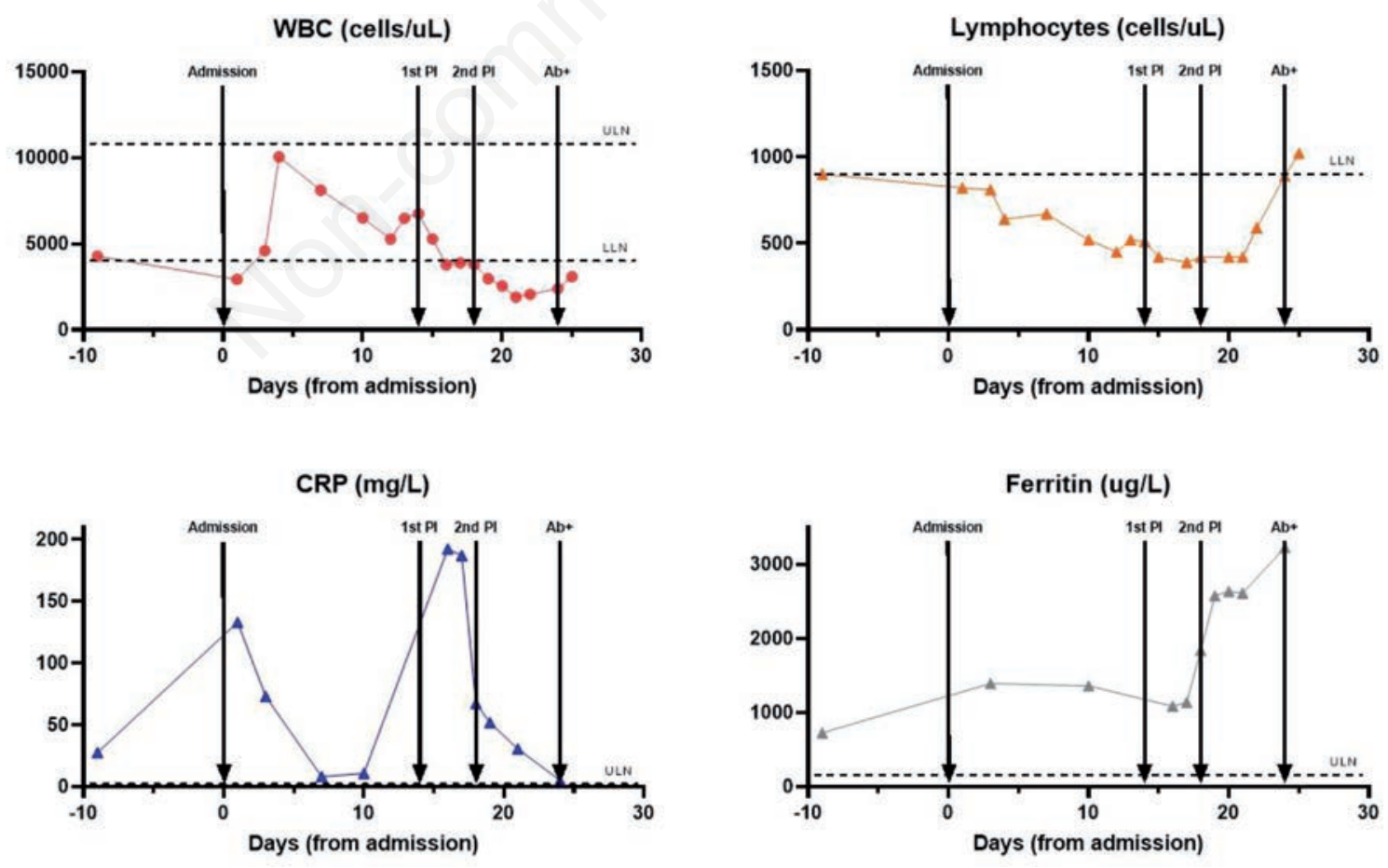

Figure 2. Main laboratory abnormalities and their trends before and during the hospital stay. WBC, white blood cells; CRP, C-reactive protein; ULN, upper limit of normality; LLN, lower limit of normality; PI, plasma infusion; Ab+, positive antibody test (IgG and IgM nucleocapsid antibodies). 
study in 105 patients with cancer and 536 age-matched controls showed that patients with cancer were at higher risk for Covid-19 and presented a higher frequency of severe events. Moreover, patients with hematologic malignancies were noted to have relatively higher death rates, intensive care unit admission rates and invasive mechanical ventilation requirements [3]. Patients receiving chemoimmunotherapy as a treatment of hematological disorders usually develop prolonged, severe iatrogenic immunosuppression. Treatment often includes the anti-CD20 antibody rituximab or the anti-CD19 antibody obinutuzumab, resulting in a delayed, marked hypogammaglobulinemia and/or lymphopenia. At the end of treatment, reconstitution of innate and adaptive immunity may take several months, causing an increased risk of opportunistic infections and compromising an efficient antimicrobial response [5]. In these patients an unusual persistency of Covid-19-related symptoms and a continuous nasopharyngeal swab positivity are frequently observed [6] and may favor accelerated viral evolution [7].

The nucleotide analog remdesivir is the only antiviral agent approved for the treatment of severe Covid-19 patients to date, showing superiority to placebo in shortening the time to recovery in Covid-19 patients with evidence of lower respiratory tract infection (likely by inhibiting proliferation of viral RNA) [8], whereas the efficacy of convalescent plasma therapy is currently being assessed [4]; data on convalescent plasma for treatment of COVID-19 are still controversial but some studies suggest an improved outcome even in the late phase of the infection in immunocompetent patients [9].
Empirical treatments with hyperimmune plasma were administered during outbreaks of Ebola virus in 2014 and other viral infections, such as SARS-CoV, H5N1 avian influenza and $\mathrm{H} 1 \mathrm{~N} 1$ influenza, showing efficacy in terms of shorter hospital stay and lower mortality [5]. In a case series of 5 critically ill patients with COVID-19 and acute respiratory distress syndrome (ARDS), a possible contribution to improved clinical status has been reported after the use of hyperimmune plasma [10]. Furthermore, early administration of high-titer convalescent plasma to mildly ill infected older adults reduced the progression of Covid-19 [11].

Only few case reports in literature analyze the results of the administration of hyperimmune plasma on hematologic patients affected by Covid-19, but all of them remark the prolonged course of the disease, the severity of initial hypogammaglobulinemia and leukopenia and the rapid remission after hyperimmune plasma transfusion [12]. Bertrains et al. demonstrated that protracted Covid-19 in patients with B-cell lymphoma is associated with a lack of significant neutralizing antibody titers and impaired clearance of SARS-CoV-2 and that treatment with hyperimmune plasma resulted in an increase in neutralizing antibody titers and a clinical response [13]. In addition, Szwebel et al. analyzed the SARSCoV-2 viral load (i.e., RNAaemia) in a hematologic patient affected by Covid-19 and treated with convalescent plasma and they observed a persistently high level of RNAaemia during the whole course of the disease and a rapid negativization after the administration of hyperimmune plasma, correlated with complete clinical recovery [14].

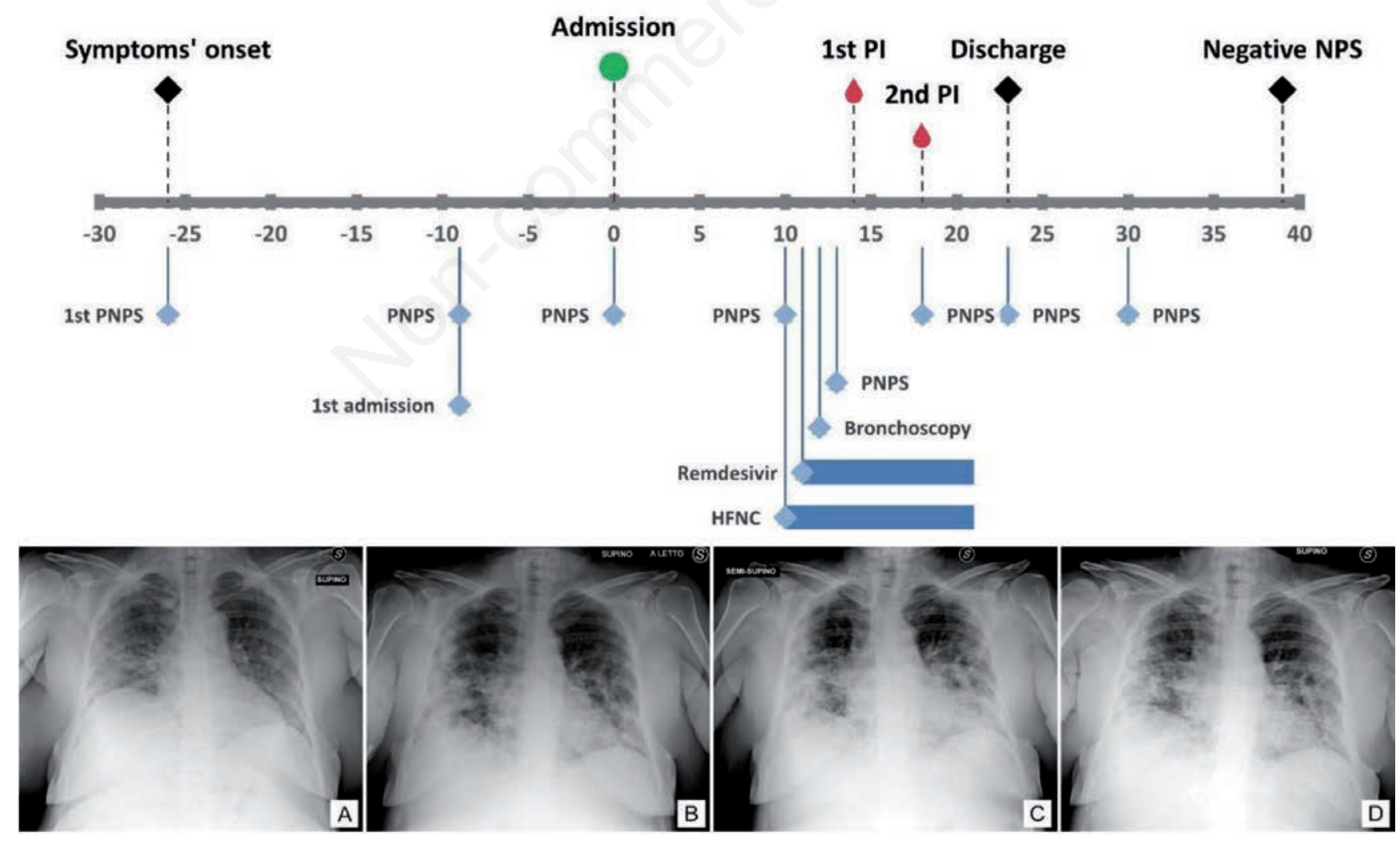

Figure 3. Upper panel: Timeline of the events. PI, plasma infusion; PNPS, positive nasopharyngeal swab; NPS, nasopharyngeal swab; HFNC, high-flow nasal cannula. Lower panel: main chest X-rays performed during the hospitalization: A) before plasma infusion (+12 days); B) after first plasma infusion (+14 days); C) right before the second plasma infusion (+18 days); D) at discharge (+23 days). 


\section{Conclusions}

Although controversial evidence currently exists for use of hyperimmune plasma for immunocompetent Covid-19 patients, very limited data are currently available in immunocompromised patients. Our case report, in agreement with the available literature, demonstrates the persistence of viral infection in a hematologic patient, due to the underlying condition of immunosuppression, and the benefit of the administration of hyperimmune plasma. As reported in other case reports, hyperimmune plasma can be a useful treatment in B-cell depleted patients and prolonged Covid-19 positivity, but additional studies are needed in order to confirm these observations.

\section{References}

1. Zhu N, Zhang D, Wang W, et al. A novel coronavirus from patients with pneumonia in China, 2019. N Engl J Med 2020;382:727-3

2. Karataş A, İnkaya AÇ, Demiroğlu H, et al. Prolonged viral shedding in a lymphoma patient with COVID-19 infection receiving convalescent plasma. Transfus Apher Sci 2020;59:102871.

3. Dai M, Liu D, Liu M, et al. Patients with cancer appear more vulnerable to SARS-CoV-2: A multicenter study during the COVID-19 outbreak. Cancer Discov 2020;10:783-91.

4. Malsy J, Veletzky L, Heide J, et al. Sustained response after remdesivir and convalescent plasma therapy in a B-cell depleted patient with protracted COVID-19. Clin Infect Dis 2020:ciaa1637. Online ahead of print.

5. Ferrari S, Caprioli C, Weber A, et al. Convalescent hyperim- mune plasma for chemo- immunotherapy induced immunodeficiency in COVID-19 patients with hematological malignancies. Leuk Lymphoma 2021;62:1490-6.

6. Shah V, Ko Ko T, Zuckerman M, et al. Poor outcome and prolonged persistence of SARS- CoV-2 RNA in COVID-19 patients with hematological malignancies; King's College Hospital experience. Br J Haematol 2020;190:e279-282.

7. Choi B, Choudhary MC, Regan J, et al. Persistence and evolution of SARS-CoV-2 in an immunocompromised host. N Engl J Med 2020;383:2291-3.

8. Beigel JH, Tomashek KM, Dodd LE, et al. Remdesivir for the treatment of Covid-19 - Final Report. N Engl J Med 2020;383:1813-26.

9. Duan K, Liu B, Li C, et al. Effectiveness of convalescent plasma therapy in severe COVID- 19 patients. Proc Natl Acad Sci USA 2020;117:9490-6.

10. Shen C, Wang Z, Zhao F, et al. Treatment of 5 critically ill patients with COVID-19 with convalescent plasma. JAMA 2020;323:1582-9.

11. Libster R, Pérez Marc G, Wappner D, et al. Early high-titer plasma therapy to prevent severe Covid-19 in older adults. $\mathrm{N}$ Engl J Med 2021;384:610-8.

12. Wright Z, Bersabe A, Eden R, et al. Successful use of COVID19 convalescent plasma in a patient recently treated for follicular lymphoma. Clin Lymphoma Myeloma Leuk 2021;21:66-8.

13. Betrains A, Godinas L, Woei-A-Jin FJSH, et al. Convalescent plasma treatment of persistent severe acute respiratory syndrome coronavirus-2 (SARS-CoV-2) infection in patients with lymphoma with impaired humoral immunity and lack of neutralising antibodies. Br J Haematol 2021;192:1100-5.

14. Szwebel TA, Veyer D, Robillard N, et al. Usefulness of plasma SARS-CoV-2 RNA quantification by droplet-based digital PCR to monitor treatment against COVID-19 in a B- cell lymphoma patient. Stem Cell Rev Rep 2021;17:296-9. 